\title{
Filling a box with translates of two bricks
}

\author{
Mihail N. Kolountzakis* \\ School of Mathematics, \\ Georgia Institute of Technology, \\ 686 Cherry Street NW, \\ Atlanta, GA 30332, USA \\ and \\ Department of Mathematics, \\ University of Crete, \\ Knossos Ave., \\ 71409 Iraklio, Greece.
}

E-mail: kolount@member . ams . org

Received: Sep 20, 2004; Accepted: Dec 1, 2004; Published: Dec 7, 2004

Mathematics Subject Classifications: 05B45, 52C22

\begin{abstract}
We give a new proof of the following interesting fact recently proved by Bower and Michael: if a $d$-dimensional rectangular box can be tiled using translates of two types of rectangular bricks, then it can also be tiled in the following way. We can cut the box across one of its sides into two boxes, one of which can be tiled with the first brick only and the other one with the second brick. Our proof relies on the Fourier Transform. We also show that no such result is true for translates of more than two types of bricks.
\end{abstract}

Suppose we have at our disposal two types of $d$-dimensional rectangles (bricks), type A with dimensions $\left(a_{1}, \ldots, a_{d}\right)$ and type $\mathrm{B}$ with dimensions $\left(b_{1}, \ldots, b_{d}\right)$. We want to use translates of such bricks to fill completely, and with no overlaps (except at the boundaries of the bricks), a given $d$-dimensional rectangular box. We then say that these two bricks tile our box by translations. All rectangles that appear in this note are axis-aligned.

Bower and Michael [1] recently showed the following nice result. A hyperplane cut is a separation of an axis-aligned box in $d$ dimensions using a hyperplane of the type $x_{j}=\alpha$,

*Supported in part by European Commission IHP Network HARP (Harmonic Analysis and Related Problems), Contract Number: HPRN-CT-2001-00273 - HARP. 
for some $j=1, \ldots, d$ and some $\alpha \in \mathbb{R}$. A hyperplane cut separates such a box into two rectangular boxes.

Theorem 1 (Bower and Michael [1]) If two bricks, of types $A$ and $B$, tile a box $Q$ (in dimension $d \geq 1$ ) by translations then we can split $Q$ into two other boxes $Q_{a}$ and $Q_{b}$ using a hyperplane cut, such that $Q_{a}$ can be tiled using translates of type $A$ bricks only and $Q_{b}$ can be tiled using translates of type $B$ bricks only.

(For $d=1$ the result is obvious.)

The purpose of this note is to give a short proof of this fact using the Fourier Transform, a very natural tool for this problem, as will become apparent. The reader could consult [2] for more applications of the Fourier Transform to tiling problems.

Indeed, suppose that $A=\left(-a_{1} / 2, a_{1} / 2\right) \times \cdots \times\left(-a_{d} / 2, a_{d} / 2\right)$ and $B=\left(-b_{1} / 2, b_{1} / 2\right) \times$ $\cdots \times\left(-b_{d} / 2, b_{d} / 2\right)$ are the two bricks and $\Lambda_{a}, \Lambda_{b}$ are two finite subsets of $\mathbb{R}^{d}$ which represent the translations of $A$ and $B$ that make up our box $Q=(-1 / 2,1 / 2)^{d}$ (as we may clearly assume without loss of generality). In other words, the indicator functions $\chi_{A}$ and $\chi_{B}$ of the two bricks satisfy

$$
\sum_{\lambda \in \Lambda_{a}} \chi_{A}(x-\lambda)+\sum_{\lambda \in \Lambda_{b}} \chi_{B}(x-\lambda)=\chi_{Q}(x), \text { a.e. } x \in \mathbb{R}^{d}
$$

The definition of the Fourier Transform $\widehat{f}$ of a function $f \in L^{1}\left(\mathbb{R}^{d}\right)$ that we use is

$$
\widehat{f}(\xi)=\int_{\mathbb{R}^{d}} f(x) \exp (-2 \pi i \xi \cdot x) d x
$$

where, as usual, $\xi \cdot x$ denotes the inner product of the vectors $\xi$ and $x$ of $\mathbb{R}^{d}$. Taking the Fourier Transform of both sides of (1) we get

$$
\phi_{a}(\xi) \widehat{\chi_{A}}(\xi)+\phi_{b}(\xi) \widehat{\chi_{B}}(\xi)=\widehat{\chi_{Q}}(\xi)
$$

where $\phi_{a}(\xi)=\sum_{\lambda \in \Lambda_{a}} \exp (2 \pi i \lambda \cdot x)$ and $\phi_{b}(\xi)=\sum_{\lambda \in \Lambda_{b}} \exp (2 \pi i \lambda \cdot x)$ are trigonometric polynomials. Simple calculation shows that the Fourier Transform of the indicator function of the box $C=\left(-c_{1} / 2, c_{1} / 2\right) \times \cdots \times\left(-c_{d} / 2, c_{d} / 2\right)$ is

$$
\widehat{\chi_{C}}(\xi)=\prod_{j=1}^{d} \frac{\sin \left(c_{j} \xi_{j}\right)}{\xi_{j}}
$$

whose zero set $Z\left(\widehat{\chi_{C}}\right)$ consists of all points $\xi$ with at least one coordinate $\xi_{j}$ being a nonzero multiple of $c_{j}^{-1}$. This set may be viewed as a collection of $d$ sets of hyperplanes, with the hyperplanes in the $j$-th set being parallel to the hyperplane $\xi_{j}=0$ and spaced at regular intervals $c_{j}^{-1}$, with the exception of the hyperplane $\xi_{j}=0$ itself (see Figure 1 ).

Therefore the zero set of the right hand side of (2) is the set

$$
Z=Z\left(\widehat{\chi_{Q}}\right)=\left\{\xi \in \mathbb{R}^{d}: \xi_{j} \in \mathbb{Z} \backslash\{0\} \text {, for some } j=1, \ldots, d\right\} \text {. }
$$




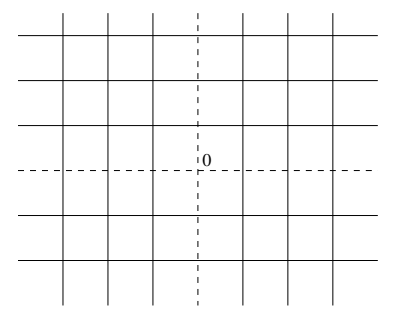

Figure 1: The zeros (solid lines) of the Fourier Transform of a rectangle in 2 dimensions

The key observation is the following: for any choice of different $i$ and $j$ from the numbers $1, \ldots, d$ at least one of $a_{i}^{-1}$ and $b_{j}^{-1}$ is an integer. For, assuming otherwise, the hyperplanes $\xi_{i}=a_{i}^{-1}$ and $\xi_{j}=b_{j}^{-1}$ would be part of the zero sets of the first and second terms in the left hand side of (2) respectively. But the intersection of these hyperplanes, on which set the left hand side vanishes, contains points not in the set $Z$ of (4), a contradiction.

Finally, if the numbers $a_{1}^{-1}, \ldots, a_{d}^{-1}$ are all integers, then brick $A$ can tile $Q$ alone, and there is nothing to prove. So we may assume that one of them is not an integer, say $a_{1}^{-1} \notin \mathbb{Z}$. By choosing $i=1$ and $j=2,3, \ldots, d$ in turn, and using our key observation above, we deduce that all $b_{j}^{-1}, j=2,3, \ldots, d$, are integers. For the same reason as before we can also assume that $b_{1}^{-1}$ is not an integer (otherwise brick $B$ can tile alone), which in turn shows that all $a_{j}^{-1}, j=2,3, \ldots, d$, are integers. Hence the face of each brick parallel to the $x_{1}=0$ hyperplane can tile the corresponding face of $Q$.

On the other hand, by the assumed tiling of $Q$ by translates of bricks $A$ and $B$ it follows, by looking along the first coordinate axis, that $1=m a_{1}+n b_{1}$ for some nonnegative integers $m$ and $n$. Then partition the box $Q$ by the hyperplane $x_{1}=-1 / 2+m a_{1}$ into two boxes of dimensions $m a_{1} \times 1 \times \cdots \times 1$ and $n b_{1} \times 1 \times \cdots \times 1$. The first box can tiled by brick $A$ by simply tiling its $1 \times \cdots \times 1$ face and repeating this $m$ times. The second box can be tiled similarly by box $B$, as we had to show.

An example. Let us observe that there is no generalization of this result to three or more bricks. That is, there are boxes which admit tilings with translates of three types of bricks, but which cannot be split into two parts using a hyperplane cut so that each of these parts can be tiled by a proper subset of the available types of bricks. It is enough to give an example in two dimensions, as any such example can be transformed to one in dimension $d>2$ be considering all bricks to have their last $d-2$ coordinates equal to 1 , and considering the $d$-dimensional tiling that arises by one layer of the two-dimensional example.

To see a two-dimensional example take $R$ much larger than 1 and use the three brick types $1 \times R, R \times 1$ and $(R-1) \times(R-1)$. With these we can tile a $(R+1) \times(R+1)$ box as shown in Figure 2. But the box cannot be split into two boxes using a hyperplane cut, each of which can be tiled using a proper subset of the available brick types. This can be verified by examining the few possibilities. 

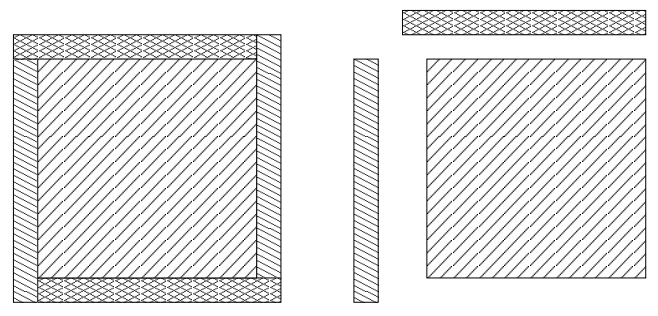

Figure 2: A tiling of a rectangle (left) with three types of bricks (right)

\section{References}

[1] R.J. Bower and T.S. Michael, When can you tile a box with translates of two given rectangular bricks?, Electr. J. Combin. 11 (2004), \#N7.

[2] M.N. Kolountzakis, The study of translational tiling with Fourier Analysis, in Fourier Analysis and Convexity, 131-187, Appl. Numer. Harmon. Anal., Birkhäuser Boston, Boston, MA, 2004. 